\title{
DUODENOPANCREATECTOMÍA COMPLETAMENTE LAPAROSCÓPICA EN EL MANEJO DEL CÁNCER PERIAMPULAR: ESTUDIO DE UN CASO
}

\author{
DUODENOPANCREATECTOMY COMPLETELY \\ LAPAROSCOPIC IN THE MANAGEMENT PERIAMPULAR CANCER: CASE STUDY \\ Arturo Orellana-Vicuñaa,b,c, Juan Tamayo-León ${ }^{b, c}$, Cesar Romero-Osoriob,c, \\ Manuel O. Huamán a, , Manuel Huamán-Guerreroa,b
}

\begin{abstract}
RESUMEN
Objetivos: La duodenopancreatectomia es una de las cirugías más complejas y desafiantes, el acercamiento laparoscópico es una alternativa a considerar. El objetivo es presentar la experiencia inicial de un paciente operado con duodenopancreatectomía completamente laparoscópica, así como los aspectos técnicos del abordaje. Métodos: Estudio prospectivo de caso único. Se analizó características del paciente, diagnóstico, técnica quirúrgica, tiempo operatorio, complicaciones intra y posoperatorias, tiempo de internación, evolución y aplicabilidad del abordaje. Resultados: Se intervino quirúrgicamente a una paciente cuyo diagnóstico final fue colangiocarcinoma, durante la disección la zona más laboriosa fue la del pedículo hepático, túnel retropancreático y proceso uncinado; la reconstrucción se realizó por medio de 3 anastomosis: pancreaticoyeyuno tipo telescopaje, hepáticoyeyuno término lateral y la gastroyeyuno término lateral. El tiempo operatorio fue de 8 horas, reportamos procedimiento de Whipple clásico completamente laparoscópico. Presentó pequeña fuga biliar, que cede al $9^{\circ}$ día, se administró somatostatina análoga cada 8 horas por 5 días. Presento arritmia cardiaca manejada clínicamente. La paciente evoluciona satisfactoriamente y en condiciones de alta 10 th día post operatorio, se queda 6 días más para manejo médico arritmia cardíaca. Conclusión: La duodenopancreatectomía totalmente laparoscópica es una técnica factible en pacientes seleccionados con tumores periampulares. Se requiere tener el paciente adecuado, el equipamiento e instrumental adecuado, el equipo quirúrgico capacitado y entrenado.
\end{abstract}

Palabras clave:Duodenopancreatectomía; Procedimiento deWhipple; Colangiocarcinoma; Laparoscopía. (fuente: DeCSBIREME)

\begin{abstract}
Objective: Duodenopancreatectomy is one of the most complex and challenging procedures, the laparoscopic approach is an alternative to take into consideration. The objective is to present the initial experience of a patient operated with Fully laparoscopic duodenopancreatectomy, as well as the technical aspects of the approach. Methods: Prospective case study. Patient characteristics, diagnosis, surgical technique, operative time, intraoperative and postoperative complications, hospitalization time, evolution and applicability of the approach were analyzed. Results: We report the procedure of a patient whose final diagnosis was cholangiocarcinoma, during the dissection the most laborious area was the hepatic pedicle, the retropancreatic tunnel and the uncinated process, the reconstruction was done by 3 anastomoses: telescoping pancreaticojejunal, termino-lateral hepatojejunal and termino-lateral gastrojejunal. The operatory time was 8 hours. We report classic Whipple's procedure completely laparoscopic. A small biliary leak appeared, which stopped the $9^{\circ}$ day, was given somatostatin analogous every 8 hours for 5 days. She presented cardiac arrhythmia the was managed clinically, the patient progresses satisfactorily In high conditions on the 10th postoperative day, he remains 6 days longer for cardiac arrhythmia. Conclusions: Fully laparoscopic duodenopancreatectomy is a feasible technique in selected patients with periampullar tumors. It requires to have the adequate patient, the appropriate equipment and instruments, the trained and trained surgical team.
\end{abstract}

Key words: Pancreatoduodenectomy; Whipple's procedure; Cholangiocarcinoma; Laparoscopy. (source: MeSH NLM)

\footnotetext{
a Facultad de Medicina, Universidad Ricardo Palma, Lima, Perú.

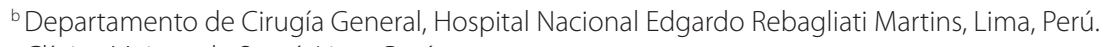

c Clínica Maison de Santé, Lima, Perú.
}

Correspondencia: Manuel Huamán Garcia. Dirección: Jirón Los Helechos 239, Urb. La molina vieja I etapa, La molina. Lima, Perú. Teléfono: (+511) 987408269. Correo : manuelhuamangarcia1@gmail.com (M.O. Huamán).

Citar como: Arturo Orellana-Vicuña, Juan Tamayo-León, Cesar Romero Osorio, Manuel O. Huamán, Manuel Huamán-Guerrero. Duodenopancreatectomía completamente laparoscópica en el manejo del cáncer periampular: Estudio de un caso [Artículo Original]. Rev. Fac. Med. Hum. 2016;16(3):13-20. DOI 10.25176/RFMH.v16.n3.646 


\section{INTRODUCCIÓN}

La duodenopancreatectomía (DP) es una cirugía técnicamente exigente que requiere largos tiempos de operatorios, disección de estructuras nobles y la construcción de anastomosis de alta complejidad'. La mortalidad postoperatoria mejoró notoriamente durante las últimas décadas de $45 \%$ a $5 \%$, sin embargo aún se asocia a alta morbilidad $(41,2 \%)$ y frecuentes estadías hospitalarias prolongadas ${ }^{1-3,9}$.

El primer caso reportado de duodenopancreatectomía laparoscópica (DPL) se acredita a Gagner y Pomp en $1992^{4}$. En Latinoamérica el primer caso reportado de DPL mano asistida le corresponde a De Vinatea et al. en $2003^{5}$. La escasa evidencia existente debida al bajo porcentaje de casos operables, el mal pronóstico a pesar de un procedimiento técnicamente satisfactorio y la extensa curva de aprendizaje que requiere limita uso de la técnica mínimamente invasiva en la práctica clínica'. Las revisiones sistemáticas realizadas en los últimos años no demuestran superioridad sobre la técnica abierta en tiempo operatorio, trauma operatorio, aparición de fistula y tiempo de recuperación postoperatorio ${ }^{1,6-10}$.

Actualmente se considera que la técnica mínimamente invasiva es un equivalente a la técnica abierta siempre que sea ejecutado por cirujanos experimentados en un centro de alta incidencia referencial para el tratamiento de las neoplasias coledocoduodenopancreáticas ${ }^{7,8}$.

Se presenta según nuestro conocimiento el primer caso reportado en la literatura peruana de duodenopancreatectomía totalmente laparoscópica. El objetivo del presente trabajo es presentar la experiencia inicial de un paciente operado con DPL, así como los aspectos técnicos del abordaje.

\section{MÉTODOS}

Estudio prospectivo de caso único. Se analizó características del paciente, diagnóstico, técnica quirúrgica, tiempo operatorio, complicaciones intra y posoperatorias, tiempo de internación, evolución y aplicabilidad del abordaje. El estudio se realizó cumpliendo los requerimientos éticos institucionales, la paciente firmó su consentimiento informado, para recibir la intervención por vía laparoscópica.

\section{RESULTADOS}

\section{Estudio de caso único}

Mujer de 72 años, natural de Arequipa, procedente de Lima, sin antecedentes de importancia. Ingresa por emergencia presentando, dolor abdominal e ictericia progresiva de 1 mes de evolución. Se le realiza ecografía abdominal donde destaca la marcada dilatación de vías biliares extra e intrahepática, además se asocia a bilirrubina total de $11 \mathrm{mg} / \mathrm{dl}$ a predominio directa y marcador tumoral CA $19-9$ en $900 \mathrm{UI} / \mathrm{ml}$.
Se hospitaliza, realizando CPRE con colocación de endoprotesis biliar.

La colangioresonancia mostró lesión neoformativa de $3 \mathrm{~cm}$ de longitud, sospechosa de neoplasia maligna (NM) a nivel del contorno cefálico y medial de la cabeza pancreática a considerar NM primario pancreático como primera posibilidad y como segunda colangiocarcinoma del colédoco distal.

El hallazgo descrito condiciona dilatación de las vías biliares, no presenta signos de compromiso infiltrativo tumoral de los vasos mesentéricos, se asocia con adenopatía tumoral en la ventana porto cava, escaso líquido libre laminar perihepático y periesplénico, la vesícula biliar distendida con barro biliar.

Lapacienteesintervenidaquirúrgicamenteel 14dediciembredel 2016, realizándose duodenopancreatectomía completamente laparoscópica, con tiempo operatorio de 8 horas (480 minutos).

La revisión anatomo-patológica de la pieza operatoria da como resultado: Adenocarcinoma tubular bien diferenciado, infiltrante de colédoco intra y extrapancreático; la neoplasia infiltra la pared del colédoco sobrepasándola e infiltrando tejido adiposo pericoledociano, no se observa invasión vascular ni perineural, bordes de sección de estómago y yeyuno libres de neoplasia, bordes de sección de colédoco y páncreas con cambios displásicos del epitelio, ampolla de Vater libre de neoplasia, con erosión y ulceración focal, ganglios peripancreáticos libres de neoplasia (0/12), el páncreas presenta extensa pancreatitis crónica severa. Estadio tumoral T2NOMx.

Luego de concluir la intervención quirúrgica la paciente es transferida a la unidad de cuidados intensivos donde permanece 4 días, presenta pequeña fuga biliar, que cede al $9^{\circ}$ día (Figura 1), se administró somatostatina análoga cada 8 horas, por 5 días. La paciente evoluciona satisfactoriamente desde el punto de vista quirúrgico y curso con arritmia cardíaca e izquemia cardíaca manejada.

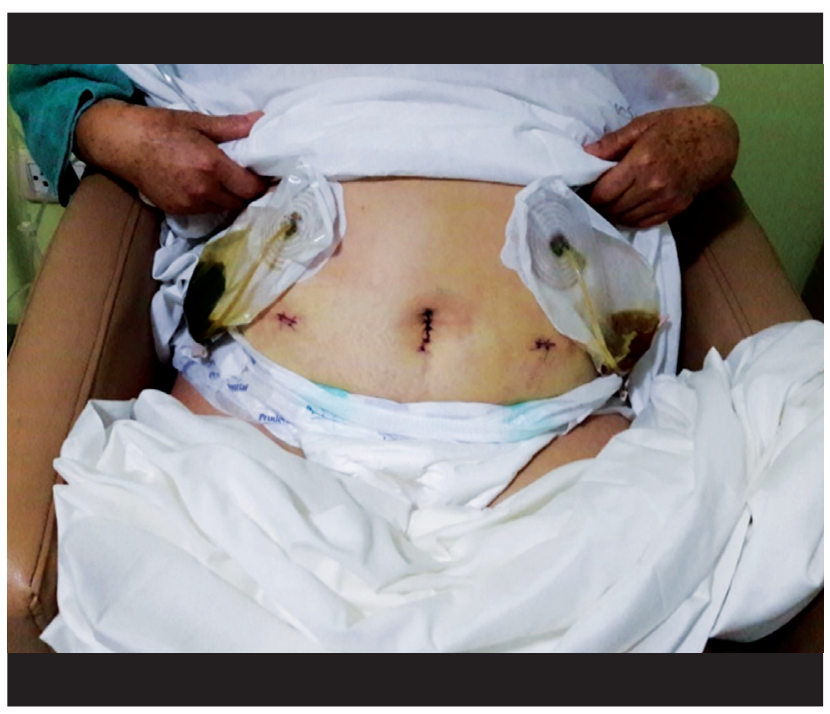

Figura 1. Paciente 7 día post operatorio, tolerando vía oral, pequeña fuga biliar. 


\section{PROCEDIMIENTO QUIRÚRGICO}

\section{ANESTESIA Y POSICIÓN DEL PACIENTE}

Realizamos el procedimiento bajo anestesia general endotraqueal, con monitoreo intraoperatorio multiparámetro. Se coloca catéter venoso central, sonda vesical y sonda nasogástrica. Se utilizó el abordaje medial, el paciente es colocado en posición de semilitotomía con ambas piernas separadas y con una ligera inclinación lateral izquierda; se realiza la antisepsia con gluconato de clorhexidina al $4 \%$, se colocan 4 campos estériles, 2 sabanas laterales estériles y 1 poncho estéril. El cirujano se ubica entre las piernas del paciente y los asistentes a cada lado del paciente.

\section{INSTRUMENTAL UTILIZADO (Tabla 1)}

Tabla 1. Instrumental utilizado durante la DPL.

\section{EQUIPO DE LAPAROSCOPIA}

- Cámara digital 3 chip image1 en alta definición

- 2 monitores, altura hombro derecho e izquierdo

- Insuflador de 30 litros por minuto

- Fuente de luz xenón 300

- Óptica de $10 \mathrm{~mm} 30$ grados

\section{INSTRUMENTAL BÁSICO}

- 2 Pinzas grasper

- 1 pinza grasper alargada y fenestrada

- 1 pinza disectora Maryland

- 2 pinzas grasper con almohadillas atraumáticas

- 2 pinzas intestinales

- 1 electrodo de hook

- 1 cánula de aspiración - irrigación

- 1 pinza bipolar

- 1 clipadora clip titanio ML

- 1 clipadora hemolok para clip de polímero ML verde

- 1 clipadora hemolok para clip de polímero L violeta

- 1 portagujas laparoscópico

- 1 tijera laparoscópica

\section{INSTRUMENTAL ESPECIAL}

- 1 disector ultrasónico (Sonicision) 5mm

- 1 Ligasure Maryland 5mm

- 1 Grapadora Echelon Flex de 60

- 2 cartuchos de Echelon dorados 60 (3.8mm x 60mm)

- 2 cartuchos de Echelon azul $60(3.5$ x 60 mm)

- 1 bolsa de extracción de espécimen

\section{TROCARES}

Realizamos neumoperitoneo abierto, luego de una incisión transumbilical colocamos trocar de $5 \mathrm{~mm}$ para realizar la insuflación, cuando tenemos $15 \mathrm{mmHg}$ de presión intraabdominal, reemplazamos trocar por uno de $10 \mathrm{~mm}$, ingresa la cámara y los demás trocares son colocados bajo visión.

- P1: 10mm, mano derecha $1^{\circ}$ ayudante, para la cámara, umbilical

- P2: 12mm, mano izquierda cirujano, línea medio clavicular derecha - umbilical

- P3: $10 \mathrm{~mm}$, mano izquierda $1^{\circ}$ ayudante, línea medio clavicular derecha - subcostal derecha

- P4: $5 \mathrm{~mm}$, mano derecha $2^{\circ}$ ayudante, subxifoideo

- P5: $10 \mathrm{~mm}$, mano izquierda $2^{\circ}$ ayudante, línea medio clavicular izquierda - subcostal izquierda

- P6: 12mm, mano derecha cirujano, línea medio clavicular izquierda - umbilical 


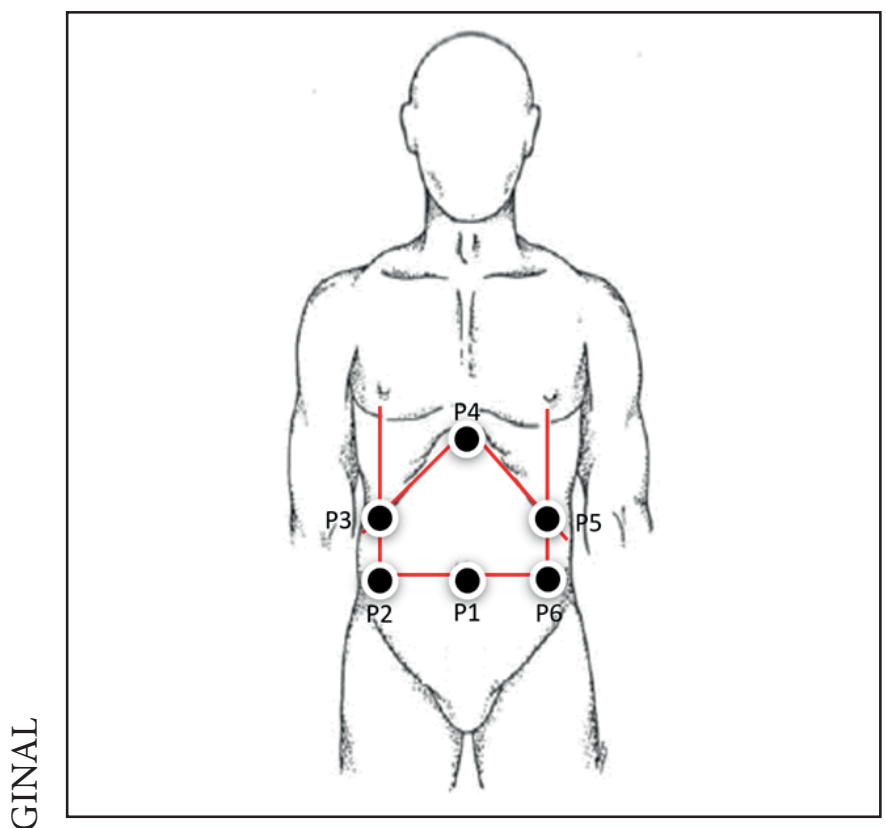

Figura 2. Ubicación de los trocares - DPL

\section{TÉCNICA OPERATORIA}

Duodenopancreatectomia clásica tiene 4 fases

1.- Estadificación y evaluación de resecabilidad

2.- Resección

3.- Reconstrucción

4.- Extracción del espécimen y revisión de drenaje

\section{ESTADIFICACIÓN Y EVALUACIÓN DE RESECABILIDAD}

Se realiza laparoscopia, el líquido libre escaso es aspirado y enviado para citología, se realiza un examen sistemático del peritoneo, ambos lóbulos hepáticos y órganos intraabdominales no se detectan lesiones; se procede a la apertura del epiplón gastrohepático utilizando el disector ultrasónico, se expone el lóbulo caudado, tronco celiaco y vena cava; apertura del epiplón gastrocólico con el disector ultrasónico para visualizar el cuerpo y la cola del páncreas. La extensión, tamaño y movilidad del tumor son evaluados indirectamente, en esta etapa contar con la ayuda de la ultrasonografía laparoscópica consideramos que sería muy importante.

\section{RESECCIÓN}

Es necesario recordar que la disección incluye 3 planos y la reconstrucción 3 estructuras (pancreáticoyeyuno, hepáticoyeyuno y gastroyeyuno anastomosis).

Durante la resección tener presente el número 4, ya que 4 vasos son ligados y seccionados (Tronco gastrocólico o vena de Henle, Arteria gastroduodenal, vasos gastroepiplóicos derechos y arteria pancreático duodenal inferior) asimismo 4 órganos son seccionados (estómago, yeyuno, conducto hepático común y páncreas).

\section{Movilización de la cabeza del páncreas}

Procedemos a descomprimir la vesícula biliar y mediante su tracción conseguimos separar el hígado para realizar la maniobra de Cattle y la maniobra de Kocher, el peritoneo colohepático es abierto, el ángulo hepático y colon transverso son movilizados hacia abajo, de esa manera queda expuesta la segunda y tercera porción del duodeno hasta el cuello del páncreas, la maniobra de Kocher se realiza traccionando el duodeno medial y anteriormente con la pinza subxifoidea, podemos visualizar riñón, vena renal, vena cava inferior, duodeno, proceso uncinado, la disección continua por encima y a lo largo de la vena cava inferior, en la parte inferior disecamos hasta la tercera porción duodeno, alcanzando el compartimiento infracólico y visualizando los vasos mesentéricos inferiores.

La sección del tronco venoso gastrocólico o vena de Henle, facilita la separación del cuello del páncreas de la vena mesentérica superior, la vena y la arteria gastroepiploica derecha son clipadas y seccionadas, se realiza sección epiplón mayor con dirección al estómago y se procede a realizar la resección del antro gástrico con Endo GIA Echelon Dorado de $60 \mathrm{~mm}$.

A continuación, se diseca el hilio hepático aperturando el peritoneo anterior y lateralmente, a la altura del conducto hepático común. Se remueve los ganglios linfáticos.

La arteria gastroduodenal es identificada entre la cabeza y cuello del páncreas, es ligada con hemolok L y seccionada. Se secciona el conducto hepático común por encima de su unión con el conducto cístico.

\section{Movilización duodeno yeyunal}

Se identifica y secciona el ligamento de Treitz visualizando la vena mesentérica inferior, a $15 \mathrm{~cm}$ el yeyuno es seccionado con Endo GIA Echelon 60 mm Azul (Figura 2), sección de vasos mesenterios del yeyunoproximal y duodeno con ligasure de $5 \mathrm{~mm}$, teniendo especial cuidado de no lesionar las pequeñas venas tributarias de la vena mesentérica superior, vena porta y del proceso uncinado cuyo sangrado podría dificultar este paso. Los extremos libres del yeyuno y duodeno son desplazados hacia el compartimiento supracólico (Figura 3). 


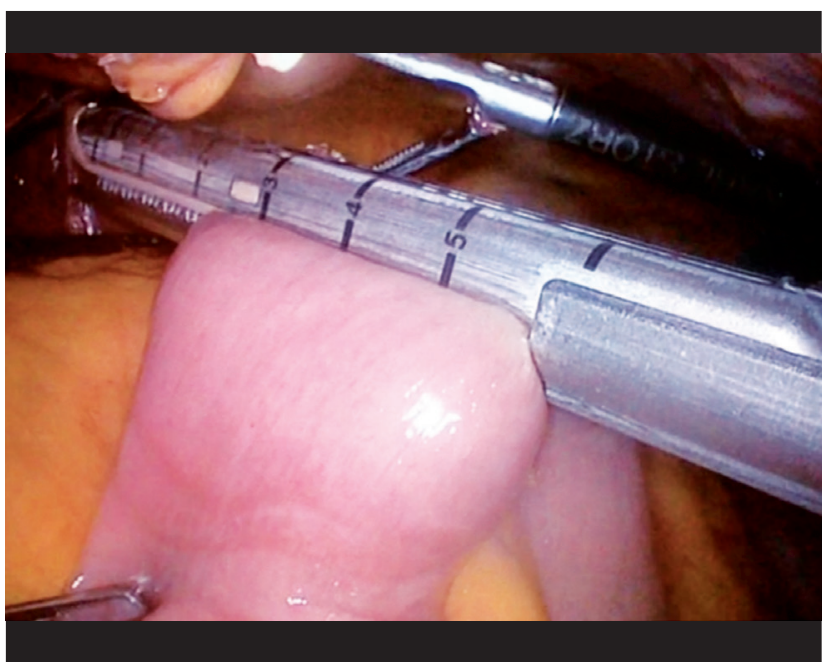

Figura 3. Sección yeyunal con ENDO-GIA.

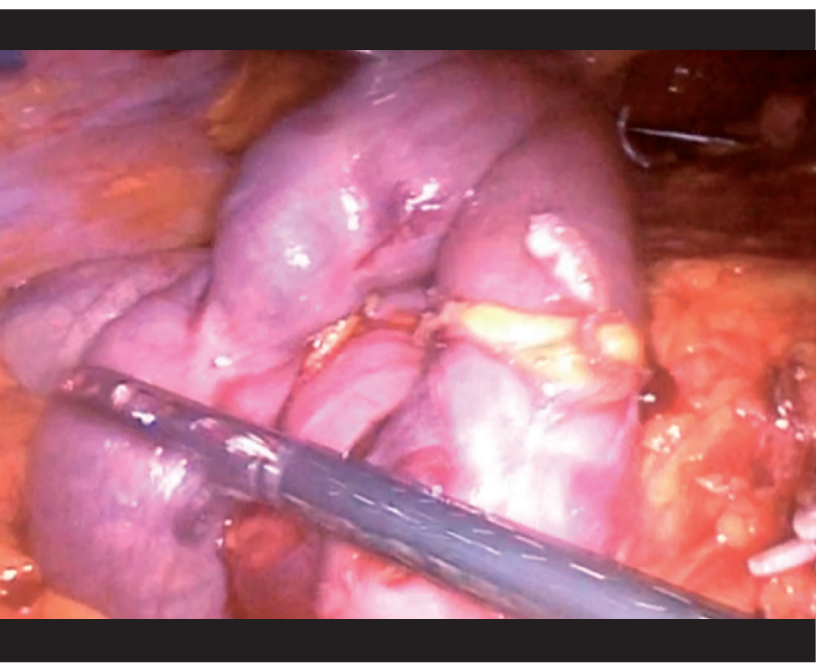

Figura 4. Duodeno y yeyuno en el compartimiento supramesocólico.

\section{Sección del Páncreas}

En este caso el túnel retropancreático fue difícil de realizar en su tramo distal, por lo tanto, decidimos sección cuidadosa del cuello del páncreas con bisturí ultrasónico (Figura 4), las tributarias laterales de la vena mesentérica superior de la cabeza del páncreas son controlados con el bisturí ultrasónico, la arteria pancreátoduodenal inferior es clipada y seccionada.

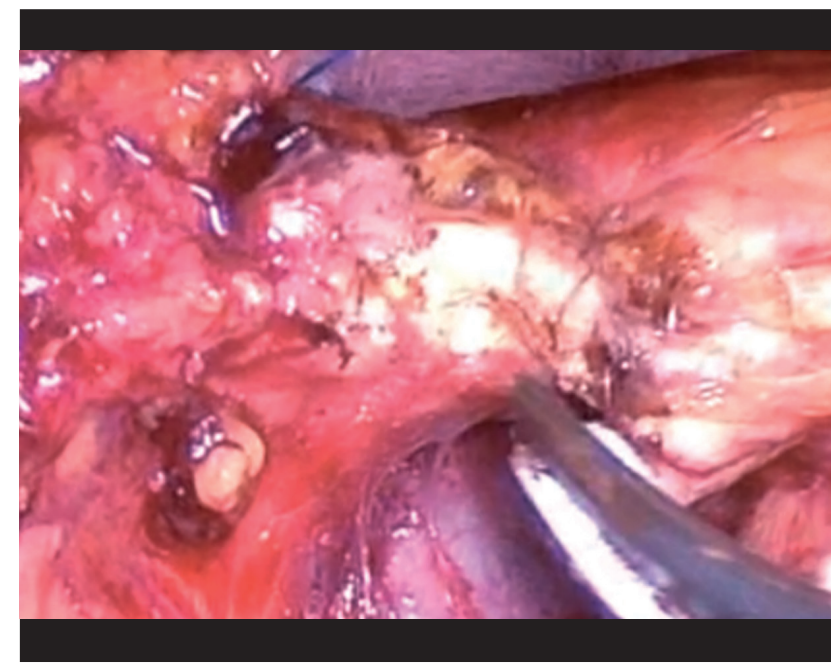

Figura 5. Sección del páncreas con bisturí ultrasónico.

\section{Resección del Proceso uncinado del páncreas}

La movilización del proceso uncinado requiere sección de vasos pequeños que se realiza con bisturí ultrasónico, las pequeñas venas tributarias del proceso uncinado y las arterias pequeñas que lo irrigan son clipadas por separado con clip de titanio ML, hemolock L (Figura 5).

Un retractor de gancho aplicado a través de la raíz del mesenterio que permite tracción anterior y a la izquierda, ayuda a la exposición del proceso uncinado.

En este paso tener mucho cuidado de no lesionar la vena porta o la vena mesentérica superior (Figura 6).

La vena que drena el páncreas se une a la vena porta en su borde superior, debe ser clipada y seccionada entre el conducto biliar y la vena porta, después de eso el espécimen queda liberado completamente y se mantiene contiguo a la zona operatoria.

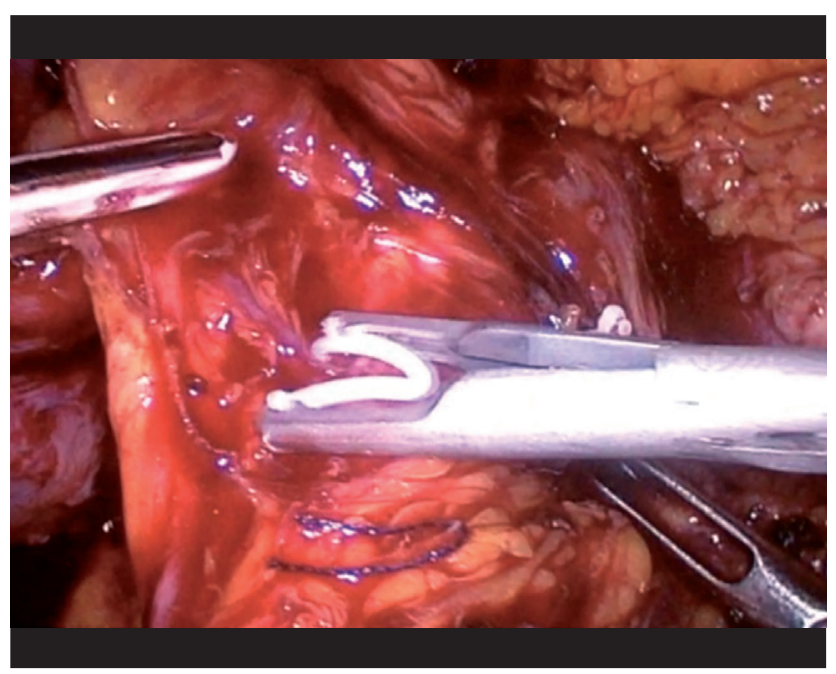

Figura 6. Disección Proceso Uncinado control vascular con Hemolock L.

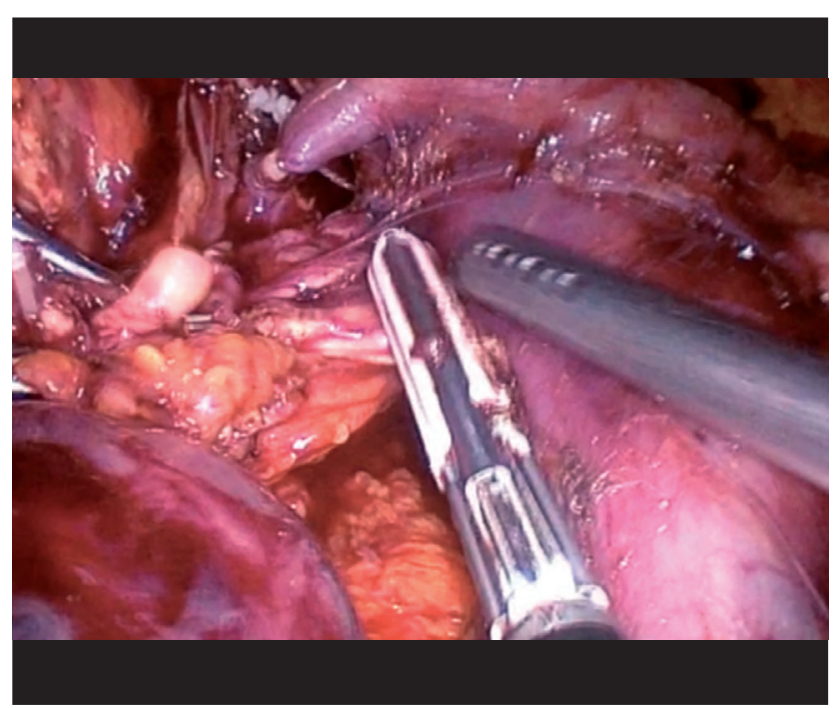

Figura 7. Disección Proceso Uncinado control vascular con ligasure. 


\section{RECONSTRUCCIÓN}

\section{Anastomosis pancreáticoyeyunal}

Observamos que el conducto de Wirsung es muy delgado e imperceptible, por ello decidimos realizar anastomosis termino terminal pancreáticoyeyunal tipo telescopaje.

Primero realizamos una ventana en el mesocolon a la derecha de la arteria cólica media, el yeyuno ingresa al compartimiento supracólico, a través de la ventana del mesocolon.

Se realiza la apertura del yeyuno proximal retirando la línea de sutura mecánica (Figura 7).

La sutura comienza desde el borde superior, la realizamos con sutura PDS 3-0, puntos separados, pancreático yeyunal (Figura 8), cara posterior y luego cara anterior, 1 solo plano (Figura 9).

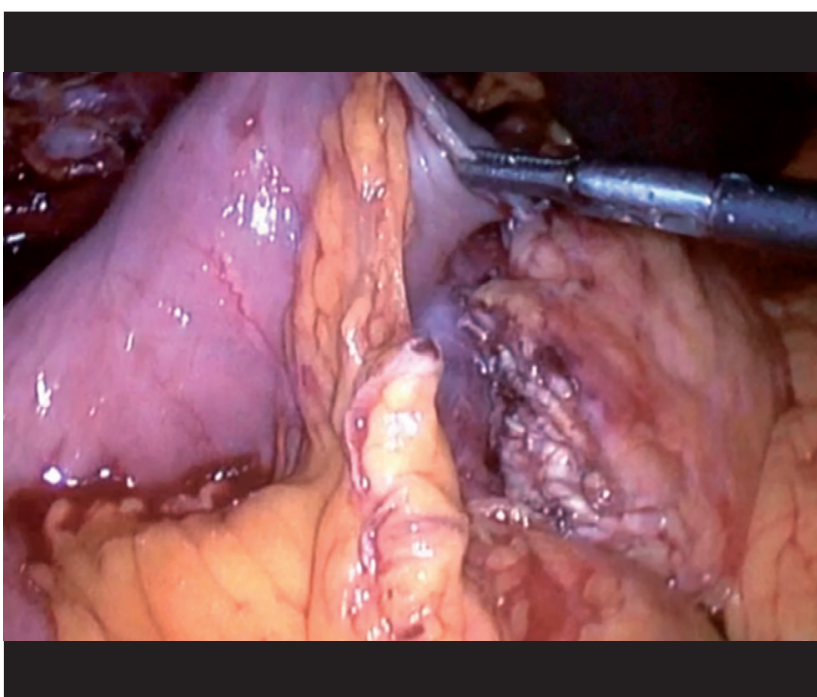

Figura 8. Apertura del yeyuno proximal retirando la línea de sutura.

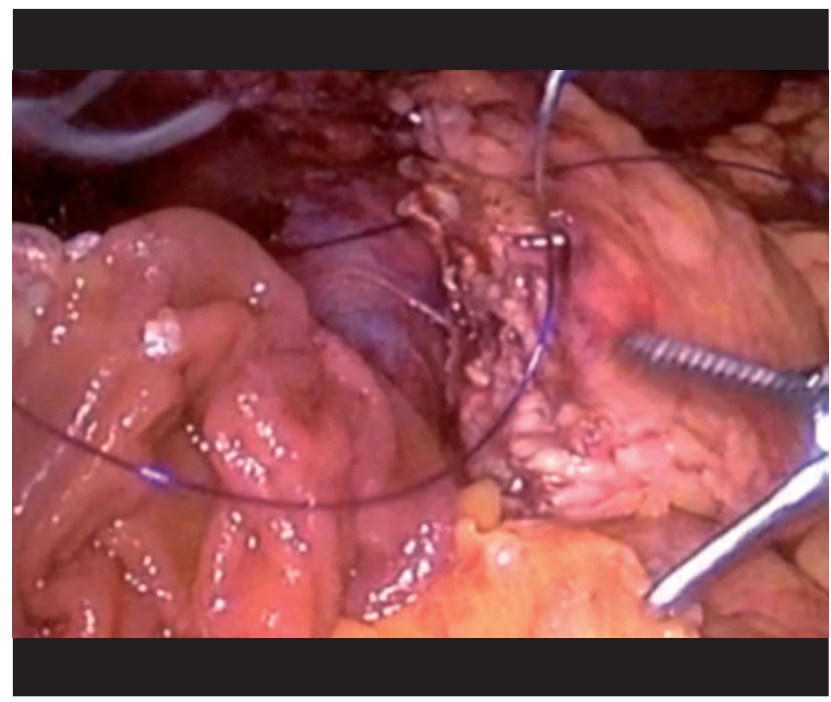

Figura 9. Sutura pancreatoyeyunal PDS 3-0 cara posterior.

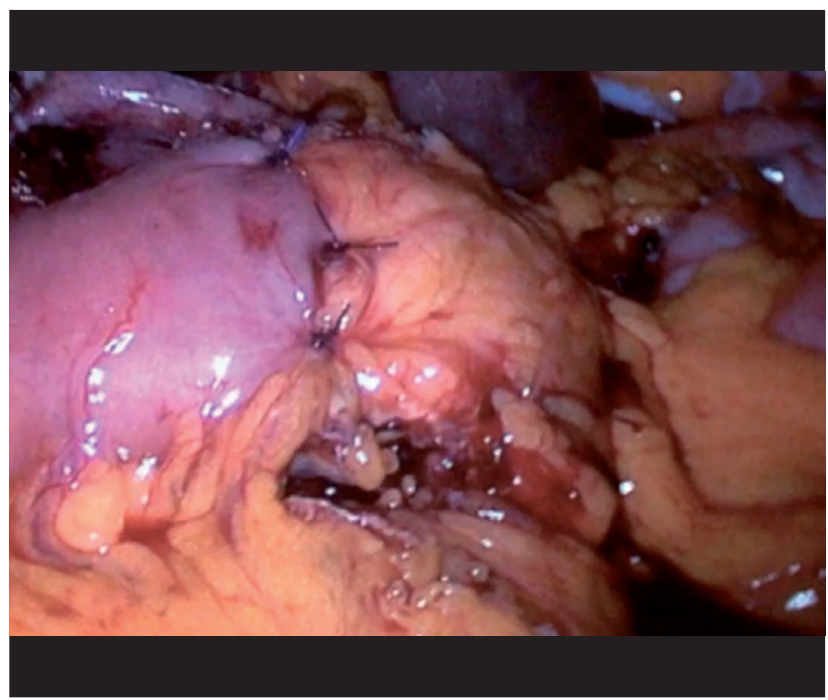

Figura 10. Anastomosis Pancreatoyeyunal tipo telescopaje.

\section{Anastomosis hepáticoyeyunal}

Observamos que el conducto hepático común tiene adecuado diámetro, decidimos realizar anastomosis hepáticoyeyunal, termino lateral (Figura 10).

Dejando yeyuno con una adecuada longitud, desde nuestra anastomosis pancreáticoyeyunal, retiramos una pequeña muesca seromuscular de yeyuno, aperturamos yeyuno con pinza Maryland, la aproximación hepáticoyeyunal se realiza con PDS 4-0 surget simple empezando de la cara posterior, 1 solo plano (Figura 11).

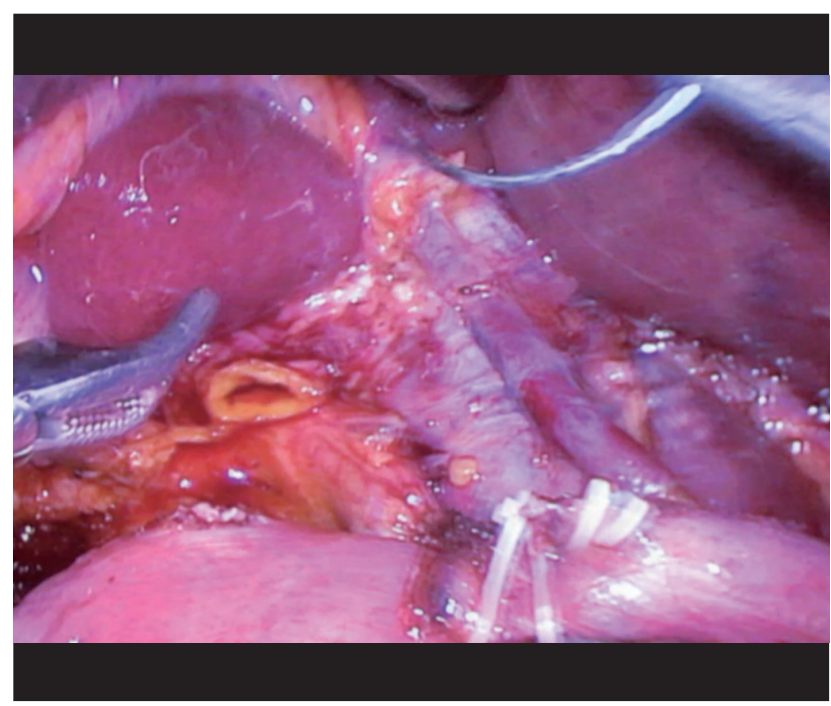

Figura 11. Anastomosis hepáticoyeyunal terminolateral.

\section{Anastomosis gastroyeyunal}

A $30 \circ 40 \mathrm{~cm}$ de la anastomosis hepáticoyeyunal, realizamos la anastomosis gastroyeyunal, términolateral.

Dejamos sin tensión anastomosis pancreáticoyeyunal y hepáticoyeyunal, ascendidendo yeyuno. En el compartimiento inframesocólico, realizamos una nueva ventana en mesocolon, llevando el asa yeyunal al compartimiento supramesocólico; con ENDOGIA 
$60 \mathrm{~mm}$ Dorado se realiza la anastomosis gastroyeyunal (Figura 12), con PDS 2.0 cerramos el orificio que deja la sutura mecánica (Figura 13).

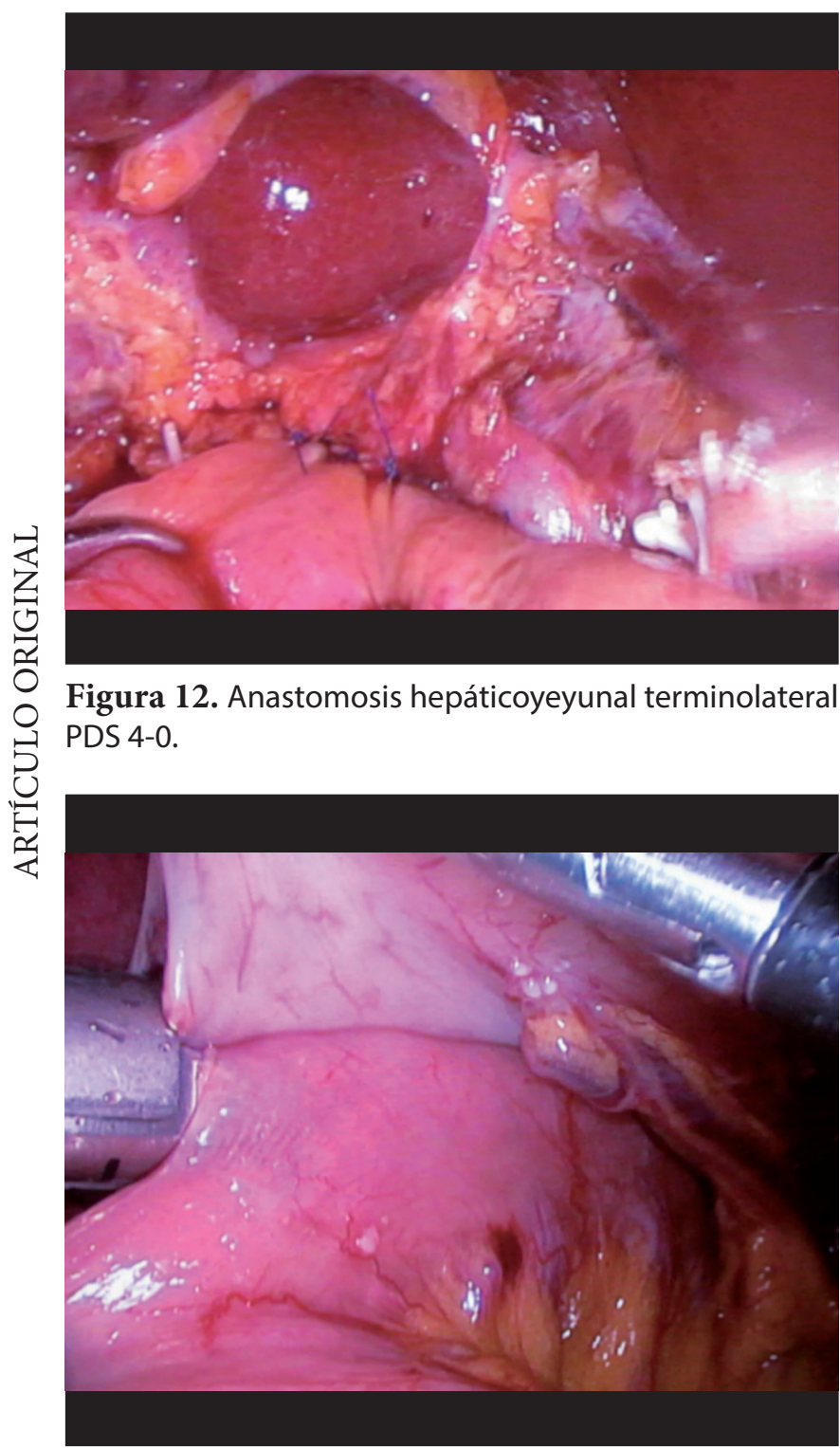

Figura 13. Anastomosis Gastroyeyunal ENDOGIA $60 \mathrm{~mm}$ x $3.8 \mathrm{~mm}$.

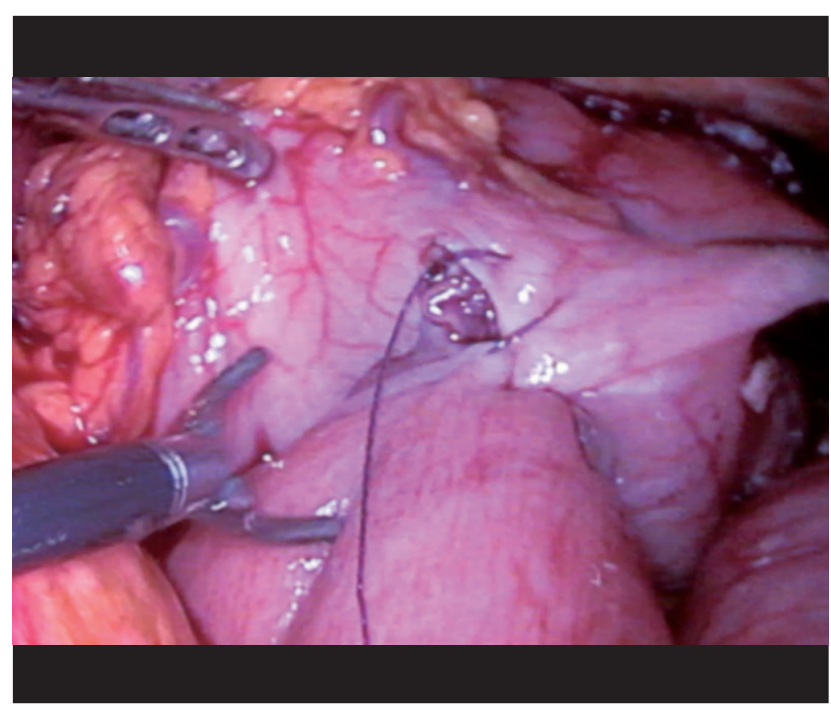

Figura 14. Cierre orificio sutura mecánica PDS 2-0-

\section{EXTRACCIÓN DEL ESPÉCIMENY REVISIÓN DEDRENAJE}

La bolsa de extracción del espécimen ingresa por trocar umbilical, depositamos toda la pieza anatómica y procedemos a retirarla, nos ayudamos con una ampliación de este puerto de $2 \mathrm{~cm}$ hacia arriba y abajo.

Se realiza la revisión de la cavidad abdominal, con especial revisión de la hemostasia y las anastomosis.

Se colocan 2 drenes tipo Jackson-Pratt en flanco derecho e izquierdo, aprovechando los orificios de los trocares de $10 \mathrm{~mm}$.

\section{DISCUSIÓN}

Se presenta los resultados de estudio de un caso de DPL realizada completamente por vía laparoscópica, el tiempo operatorio fue de 480 minutos y con una estancia hospitalaria de 10 días, resultados que se encuentran dentro de lo esperado ya que según Gagner et al. ${ }^{11}$ el tiempo operatorio promedio de la DPL estaría alrededor de 510 minutos y la estadía hospitalaria en promedio 22,3 días, asimismo Correa-Gallego et al. ${ }^{10}$ informa que el promedio podría prolongarse 130 minutos adicionales comparado con la técnica abierta. Actualmente Palanivelu et al. reporta un tiempo operatorio 370 min y una estancia hospitalaria media de 10 días, pero el estudio se aplicó solo en pacientes con puntaje ASA I y $\|^{12}$.

La respuesta inflamatoria sistémica en el periodo postoperatorio precoz se ha referido que suele ser menor para el grupo laparoscópico, lo cual se traduce en la reducción de las complicaciones postoperatorias, recuperación rápida del paciente, mejor calidad de vida y mejor tolerancia al tratamiento adyuvante ${ }^{13,14}$.

La pancreaticoyeyuno anastomosis se realizó mediante la técnica de telescopaje, y no se presentó fistula pancreática que es una de las complicaciones más peligrosas de los pacientes sometidos a DPL. Cho et al. considera a este método factible y seguro para realizar la DPL pura con una tasa de fistula del $25 \%$ similar al encontrado en la revisión de Boggi et al. de 22,3\%9,15.

El número de ganglios linfáticos resecados en la DPL es más alto que en la técnica abierta, se calcula hasta en 6 ganglios adicionales respecto a la técnica abierta ${ }^{10,16}$. En nuestro caso se resecaron 12 ganglios peripancreáticos, todos libres de enfermedad.

La DPL es una cirugía segura, factible de ejecutarla, con evidencia de que los resultados oncológicos serían semejantes a la cirugía abierta en pacientes seleccionados adecuadamente $\mathrm{e}^{1,6-10,12,14}$.

El éxito de la duodenopancreatectomia completamente laparoscópica depende de la elección del paciente adecuado, disposición del equipamiento e instrumental optimo; y, lo más importante, un equipo quirúrgico de gran experiencia en este tipo de cirugía, capacitado y entrenado en la práctica de la cirugía mínimamente invasiva. 


\section{CONCLUSIÓN}

Laduodenopancreatectomíatotalmentelaparoscópica es una técnica factible en pacientes seleccionados con tumores periampulares. Se requiere tener el paciente adecuado, el equipamiento e instrumental adecuado, el equipo quirúrgico capacitado y entrenado.
Financiamiento: Autofinanciado.

Conflicto de interés: Los autores declaran no tener conflictos de interés en la publicación de este artículo.

Recibido: 29 de diciembre del 2016

Aprobado: 16 de enero del 2017

\section{REFERENCIAS BIBLIOGRÁFICAS}

1. Al-Taan OS, Stephenson JA, Briggs C, Pollard C, Metcalfe MS, Dennison AR. Laparoscopic pancreatic surgery: a review of present results and future prospects. HPB. 2010 May;12(4):239-43.

2. Fernández-del Castillo C, Morales-Oyarvide V, McGrath D,

Wargo JA, Ferrone CR, Thayer SP, et al. Evolution of the Whipple procedure at the Massachusetts General Hospital. Surgery. 2012 Sep;152(3 Suppl 1):S56-63.

3. Herter FP, Cooperman AM, Ahlborn TN, Antinori C. Surgical experience with pancreatic and periampullary cancer. Ann Surg. 1982 Mar;195(3):274-81.

4. Gagner M, Pomp A. Laparoscopic pylorus-preserving pancreatoduodenectomy. Surg Endosc. 1994;8:408-10.

5. De Vinatea J, Villanueva L, Gonzales E. Duodenopancreatectomía laparoscópica mano asistida: Reporte de un caso. An Fac Med Lima 2004. 2004;65(3):199-202.

6. Doula C, Kostakis ID, Damaskos C, Machairas N, Vardakostas DV Feretis $\mathrm{T}$, et al. Comparison Between Minimally Invasive and Open Pancreaticoduodenectomy: A Systematic Review. Surg Laparosc Endosc Percutan Tech. 2016 Feb;26(1):6-16

7.Place TL, Nau P, Mezhir JJ. Minimally Invasive Pancreatectomy for Cancer: A Critical Review of the Current Literature. Journal of Gastrointestinal Surgery. 2015 Feb;19(2):375-86.

8. Ammori BJ, Ayiomamitis GD. Laparoscopic pancreaticoduodenectomy and distal pancreatectomy: a UK experience and a systematic review of the literature. Surg Endosc. 2011 Jul;25(7):2084-99.
9. Boggi U, Amorese G, Vistoli F, Caniglia F, De Lio N, Perrone V, et al. Laparoscopic pancreaticoduodenectomy: a systematic literature review. Surg Endosc. 2015 Jan;29(1):9-23.

10. Correa-Gallego C, Dinkelspiel HE, Sulimanoff I, Fisher S, Viñuela EF, Kingham TP, et al. Minimally-Invasive vs Open Pancreaticoduodenectomy: Systematic Review and Meta-Analysis. J Am Coll Surg. 2014 Jan;218(1):129-39.

11.Gagner M,Pomp A.Laparoscopic pancreatic resection: Is it worthwhile? J Gastrointest Surg Off J Soc Surg Aliment Tract. 1997 Feb;1(1):20-25-26.

12. Palanivelu C, Shetty R, Jani K, Sendhilkumar K, Rajan PS, Maheshkumar GS. Laparoscopic distal pancreatectomy: results of a prospective non-randomized study from a tertiary center. Surg Endosc. 2007 Mar;21(3):373-7.

13. Kondo N, Clark CJ, Que FG, Lombardo KMR, Nagorney DM, Donohue $\mathrm{JH}$, et al. Incidence of Systemic Inflammatory Response Syndrome After Total Laparoscopic Pancreatoduodenectomy - a Comparison With Open Pancreatoduodenectomy. Gastroenterology. 2012 May 1;142(5):S-1043.

14. Kendrick ML. Laparoscopic and robotic resection for pancreatic cancer. Cancer J. 2012 Dec;18(6):571-6.

15. Cho A, Yamamoto $H$, Kainuma $O$, Muto $Y$, Park $S$, Arimitsu $H$, et al. Performing simple and safe dunking pancreaticojejunostomy using mattress sutures in pure laparoscopic pancreaticoduodenectomy. Surg Endosc. 2014 Jan;28(1):315-8.

16.AsbunHJ,StaufferJA.Laparoscopicvsopenpancreaticoduodenectomy: overall outcomes and severity of complications using the Accordion Severity Grading System. J Am Coll Surg. 2012 Dec;215(6):810-9.

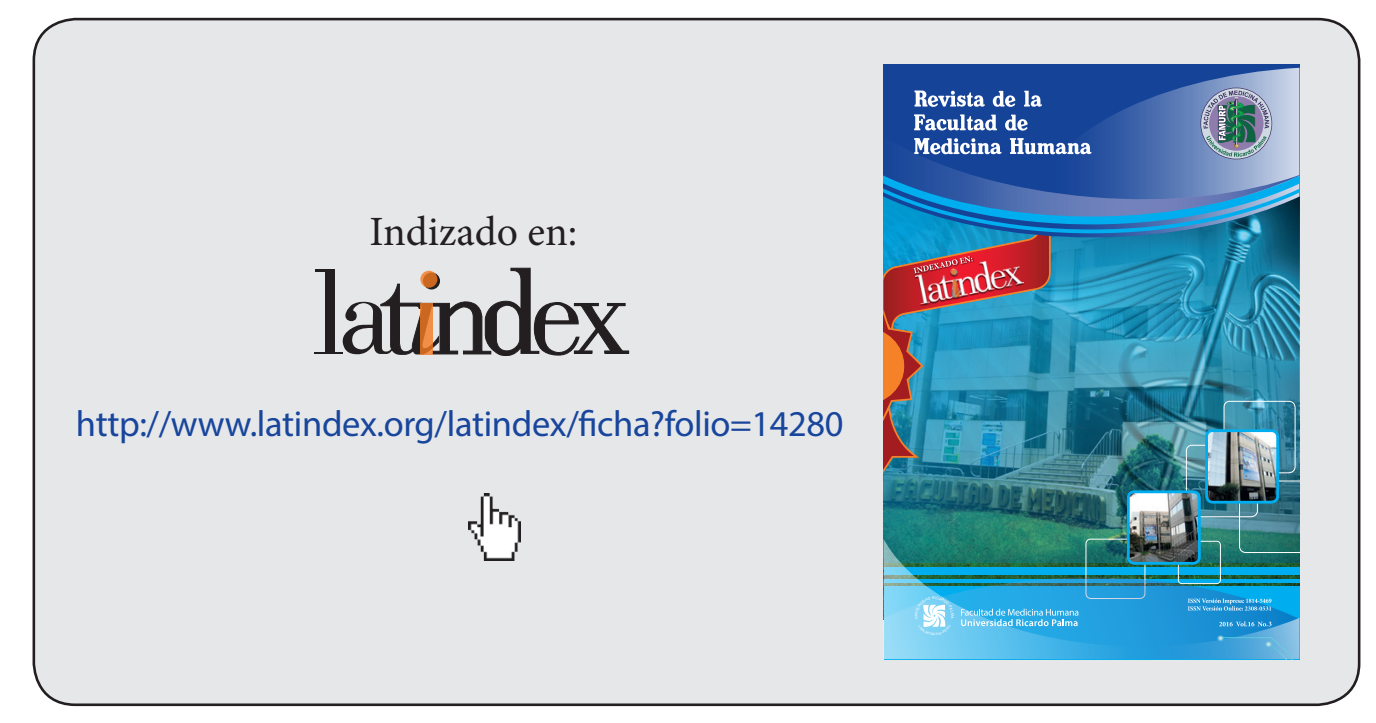

